\title{
Kinetics of Huperzine A Dissociation from Acetylcholinesterase via Multiple Unbinding Pathways
}

\author{
J. Rydzewski, ${ }^{* \dagger}$, R. Jakubowski, ${ }^{\dagger, \dagger}$ W. Nowak, ${ }^{\dagger}$ and H. Grubmüller ${ }^{\S}$ \\ ${ }^{\dagger}$ Institute of Physics, Faculty of Physics, Astronomy and Informatics, Nicolaus Copernicus University, Grudziadzka 5, 87-100 Torun, \\ Poland \\ ${ }^{\ddagger}$ Centre of New Technologies, University of Warsaw, Banacha 2c, 02-097 Warsaw, Poland \\ ${ }^{\S}$ Max Planck Institute for Biophysical Chemistry, Am Faßberg 11, 37077 Göttingen, Germany
}

Supporting Information

ABSTRACT: The dissociation of huperzine A (hupA) from Torpedo californica acetylcholinesterase ( TcAChE) was investigated by $4 \mu \mathrm{s}$ unbiased and biased all-atom molecular dynamics (MD) simulations in explicit solvent. We performed our study using memetic sampling (MS) for the determination of reaction pathways (RPs), metadynamics to calculate free energy, and maximum-likelihood estimation (MLE) to recover kinetic rates from unbiased MD simulations. Our simulations suggest that the dissociation of hupA occurs mainly via two RPs: a front door along the axis of the active-site gorge (pwf) and through a new transient side door (pws), i.e., formed by the $\Omega$-loop (residues 67-94 of TcAChE). An analysis of the
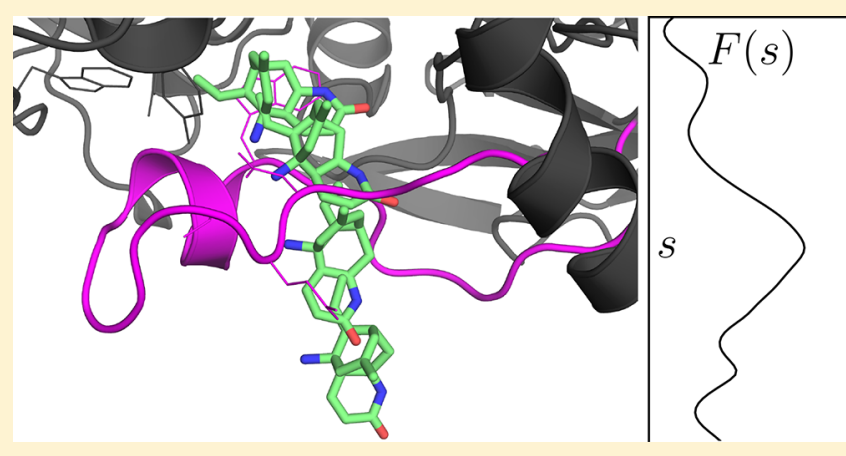
inhibitor unbinding along the RPs suggests that pws is opened transiently after hupA and the $\Omega$-loop reach a low free-energy transition state characterized by the orientation of the pyridone group of the inhibitor directed toward the $\Omega$-loop plane. Unlike pws, pwf does not require large structural changes in TcAChE to be accessible. The estimated free energies and rates agree well with available experimental data. The dissociation rates along the unbinding pathways are similar, suggesting that the dissociation of hupA along pws is likely to be relevant. This indicates that perturbations to hupA-TcAChE interactions could potentially induce pathway hopping. In summary, our results characterize the slow-onset inhibition of TcAChE by hupA, which may provide the structural and energetic bases for the rational design of the next-generation slow-onset inhibitors with optimized pharmacokinetic properties for the treatment of Alzheimer's disease.

\section{INTRODUCTION}

The main role of AChE is the termination of nerve impulse transmission at cholinergic synapses ${ }^{1}$ by rapid hydrolysis of the acetylcholine neurotransmitter. ${ }^{2}$ Its inhibition reinforces the cholinergic neurotransmission which operates at a rate approaching the diffusional limit in substrate association and dissociation. ${ }^{3,4}$ For this reason, $\mathrm{AChE}$ has been the target of many compounds ranging from organophosphorus nerve agents and insecticides to the drugs for the treatment of Alzheimer's disease ${ }^{5}$ and the recovery of neuromuscular block in surgery. ${ }^{6-8}$ As the inhibitor of AChE in clinical use, hupA has attracted considerable interest because of its lower toxicity, higher selectivity, and long time inhibition. ${ }^{9-11}$

The hupA inhibitor binds inside the catalytic anionic site (CAS) of TcAChE, located within a $20 \AA$ long and narrow gorge leading from the surface of the enzyme to the cavity containing the active site. ${ }^{12}$ The crystallographic structure of Torpedo californica AChE liganded with various inhibitors has revealed a dissociation pathway for enzymatic trafficking along the active-site gorge heading to the CAS, subsequently called the front-door pathway (pwf). There is evidence suggesting that apart from pwf, other migration channels also exist: side door (pws) and back door (pwb). It has been observed by X-ray that TcAChE liganded with hupA exhibits a transiently formed pws in the proximity of the $\Omega$-loop (residues 67-94). ${ }^{13,14}$ This pathway has been proposed to serve as a potential channel for the flux of gaseous species and ions into the active-site gorge. ${ }^{14}$ Further, the transient opening of pwb, triggered by a gating mechanism (Trp432, Trp84, Tyr442) has been identified as the egress pathway of other ligands, e.g., aflatoxin $^{15}$ and tiocholine. ${ }^{16}$

A question that remains unanswered is how the slow-onset inhibition of TcAChE can be explained in structural, thermodynamic and kinetic terms. ${ }^{3,17}$ Slow-onset enzyme inhibitors are of interest for drug discovery because their slow dissociation from the enzymatic matrix results in sustained target occupancy leading to improved pharmacodynamics. ${ }^{18}$ The hupA-inhibited TcAChE complex dissociates at a much lower rate (up to several hours) to restore enzyme activity. This phenomenon is in contrast to the rapid on- and off-rates that characterize reversible inhibitors of $T c \mathrm{AChE}$ with similar potency, and

Received: February 16, 2018

Published: May 1, 2018 
thereby hupA is assumed to have a longer residence time than most inhibitors. The reason for the unusual unbinding of hupA from TcAChE has not been provided by experiment so far.

Therefore, despite the progress in studies on AChE liganded with various inhibitors, the following aspects require analysis. (1) How does hupA leave the active-site gorge of AChE and what are the RPs of such a process? (2) What is the structural, thermodynamic and kinetic basis of the slow-onset inhibition of AChE? To address these questions, here we use methods for studying enzymatic trafficking. ${ }^{19,20}$ These methods comprise a recent memetic sampling (MS) to compute the RPs of ligand dissociation, ${ }^{21}$ metadynamics ${ }^{22}$ and maximum-likelihood estimation (MLE) $)^{23}$ to compute kinetic rates by combining results from unbiased $\mathrm{MD}$ simulations and free-energy estimates. MS has been successfully applied to identify several nonlinear $\mathrm{RP}$ of ligand dissociation, ${ }^{21,24,25}$ and metadynamics has been used to sample ligand unbinding in various systems, ${ }^{26-32}$ yielding free-energy estimates which have been shown to agree with experimental results in many cases. For an in-depth derivation and explanation of these methods, we refer to our review. ${ }^{33}$

\section{METHODS}

II.A. System. In the crystal structure of TcAChE (PDB ID: $\left.1 E A 5^{12}\right)$, missing amino acids and loops were reconstructed by Prime. ${ }^{34}$ The parameters of hupA were initially determined by $\mathrm{CGenFF}^{35,36}$ and corrected using the Force Field Toolkit. ${ }^{37}$ Quantum mechanical calculations were performed using Gaussian $09^{38}$ and the $6-31 \mathrm{G}^{*}$ basis set to ensure compatibility with the used CHARMM force field. The geometry of hupA was optimized and partial charges were calculated according to the CHARMM standard. ${ }^{39,40}$ To reproduce interactions with TIP3P water, the hupA-TIP3P interactions were optimized by probing the positions of water molecules near possible hupA interaction sites to obtain an optimal configuration of hydrogen bonds. The hupA-TIP3P configurations were optimized using the quantum mechanical calculations, and subsequently fitted to the CHARMM force field. For the details of this procedure, see the Force Field Toolkit manual. ${ }^{37}$

The docking of hupA to $T c A C h E$ was performed using Glide. ${ }^{41}$ The resulting complex of $T c \mathrm{AChE}$ and hupA was minimized and equilibrated through $5 \mathrm{~ns}$ MD simulations under NPT conditions at $1 \mathrm{~atm}$ (the Langevin piston ${ }^{42}$ ) and $300 \mathrm{~K}$ (the Langevin thermostat) using the CHARMM22 force field ${ }^{39}$ as implemented in NAMD. ${ }^{43}$ The simulations were run in explicit solvent using the TIP3 water model ${ }^{44}$ in electrically neutralized environment $(0.15 \mathrm{~mol} / \mathrm{L} \mathrm{NaCl})$ with periodic boundary conditions. Long-range electrostatics was computed using $\mathrm{PME}^{45}$ with a $(90,90,80) \AA^{3}$ grid and a $12 \AA$ cutoff. The time step in the MD simulations was 2 fs. For a summary of the MD simulations performed in this study, we refer to Tab. S1 in the Supporting Information.

II.B. Reaction Pathways. We described the hupA-TcAChE transition from an initial state $\mathbf{R}_{A}$ to a final state $\mathbf{R}_{B}$ by a set of coordinates $\mathbf{R} \equiv\left(\boldsymbol{r}_{1}, \ldots, \boldsymbol{r}_{N}\right) \in \mathbb{R}^{3 N}$ arranged in the form of a multidimensional vector representing the $R P$, i.e., the putative reaction coordinate. The RPs of the hupA dissociation were computed using MS, an enhanced-sampling method to probe ligand dissociation from the active site of an enzyme to solvent. $^{21,24}$ The method constructs all possible heterogeneous RPs along transient tunnels without initial guesses of intermediate or final states, requiring only crystallographic information. An initial guess of the trajectory $\mathbf{R}(t)$ joining $\mathbf{R}_{A}$ and $\mathbf{R}_{B}$
(Figure 1) was determined by minimizing the effective interaction energy $\Lambda$ between hupA and the enzyme

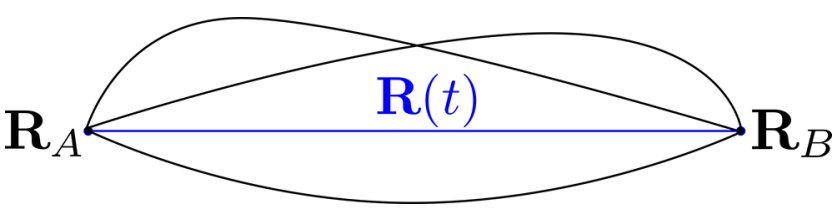

Figure 1. RP determined by an optimal dissociation trajectory $\mathbf{R}(t)$ (blue) calculated using the minimization of $\Lambda$ in MS. Unlike the trajectories shown in black, $\mathbf{R}(t)$ minimizes $\Lambda$. $\mathbf{R}(t)$ connects the initial $\mathbf{R}_{A}$ and final $\mathbf{R}_{B}$ states and provides an initial guess for the welltempered metadynamics simulations in the PCV space.

$$
\Lambda(\mathbf{R})=V\left(\mathbf{R}^{(\alpha)}, \mathbf{R}^{(\beta)}\right)+\sum_{i=1}^{N_{\alpha}} \sum_{j>i}^{N_{\beta}} h_{i j} \mathrm{e}^{-\left\|\boldsymbol{r}_{i}^{(\alpha)}-\boldsymbol{r}_{j}^{(\beta)}\right\|^{2} / 2 \sigma^{2}}
$$

which comprises the van der Waals, electrostatic and hydrogen bond interactions denoted jointly as $V$ and the term describing the conformational flexibility of hupA in the enzyme matrix. The indices $\alpha$ and $\beta$ indicate the positions of subsystems consisting of hupA and $T c A C h E$, respectively. In eq 1 , the sum is iterated over all pairs of atoms between the subsystems, where $N_{\alpha}$ and $N_{\beta}$ are the numbers of atoms in hupA and TcAChE, respectively. The width of the Gaussian is denoted as $\sigma$ and its height $h_{i j}=s_{i} v_{j}+s_{j} v_{i}$ is given by the linear combination of solvation coefficients $s$ and atomic volumes $v$.

In MS, a biasing potential (maintained during $m$ steps of an $\mathrm{MD}$ simulation) was used to guide hupA along the TcAChE tunnels. The on-the-fly procedure to determine the $k$ th intermediate configuration of hupA and $T c \mathrm{AChE}$ was the following:

1. The $k$ th hupA conformation was calculated in a neighborhood of the $(k-1)$ th hupA conformation by minimizing $\Lambda$. The neighborhood was assured by sampling the hupA conformations placed at the $(k-1)$ th conformation with a cutoff.

2. The hupA inhibitor was pulled by the biasing potential in $m$ steps of an MD simulation in the direction of the $k$ th hupA conformation.

3. After $m$ steps of an MD simulation, the conformation of the system consisting of the $k$ th hupA conformation and protein adjusted to the pulled inhibitor, is saved as the $k$ th intermediate.

Details concerning the minimization of $\Lambda$ and the sampling algorithms were described in articles by Rydzewski and Nowak. ${ }^{21,24}$ The spring connecting hupA and a virtual atom moving with constant velocity $0.05 \AA / \mathrm{ps}$ was $7.2 \mathrm{kcal} /(\mathrm{mol} \AA)^{2}$. The direction of the biasing force was recomputed every 10 ps. A cutoff of $8 \AA$ was used in sampling of the hupA conformations. To calculate the RPs, we performed 20 MS simulations of $1 \mathrm{~ns}$ duration each.

II.C. Interaction Profiling. The frequency of hydrogen bonds was profiled for each RP using the MS simulations. Hydrogen donor-acceptor pairs were identified by MDAnalysis. ${ }^{46}$ A hydrogen bond was counted between a donoracceptor pair if the distance between the atoms was less than $4.1 \AA$ and the donor-hydrogen-acceptor angle was within 100 and $180 \mathrm{deg}$. The interaction profiles of the RPs are shown in Figure S2 in the Supporting Information.

II.D. Path Collective Variables. The RPs defining the exit routes of hupA were used to perform metadynamics simulations 
in a path collective variable $(\mathrm{PCV})$ space. ${ }^{27,29,47,48} \mathrm{PCV}$ required two sets of atoms for the metadynamics simulations. The alignment set was used to superimpose RPs with the current state of the system, whereas the displacement set was employed to compute the mean-square displacement between the aligned conformations. The sets for alignment and displacement comprised of the $C_{\alpha}$ atoms of the TcAChE helices, and hupA and the $\Omega$-loop, respectively.

The RPs in the form of $\mathbf{R}(t)$ describing the transitions between the ligand-bound and ligand-unbound states calculated by MS were used to introduce two functions, $s(\mathbf{R})$ and $z(\mathbf{R}):^{29,47}$

$$
s(\mathbf{R})=\lim _{\lambda \rightarrow \infty} \frac{\int_{0}^{1} t \mathrm{e}^{-\lambda(\mathbf{R}-\mathbf{R}(t))^{2}} \mathrm{~d} t}{\int_{0}^{1} \mathrm{e}^{-\lambda(\mathbf{R}-\mathbf{R}(t))^{2}} \mathrm{~d} t}
$$

and

$$
z(\mathbf{R})=-\lim _{\lambda \rightarrow \infty} \frac{1}{\lambda} \ln \int_{0}^{1} \mathrm{e}^{-\lambda(\mathbf{R}-\mathbf{R}(t))^{2}} \mathrm{~d} t
$$

The progress along the RPs was computed by $s(\mathbf{R})$; the distance from the RPs was measured by $z(\mathbf{R})$. We parametrized the trajectories such that $\mathbf{R}(0)=\mathbf{R}_{A}$ and $\mathbf{R}(1)=\mathbf{R}_{B}$. The $\lambda$ values for the RPs are shown in Table 1 . We performed the

Table 1. Summary of the Well-Tempered Metadynamics Simulations of the RPs Representing the Pathways of the hupA Dissociation from TcAChE

$\begin{array}{ccccccc}\text { RP } & \begin{array}{c}\text { no. of } \\ \text { trajectories }\end{array} & \begin{array}{c}\text { simulation } \\ \text { time }(\mu \mathrm{s})\end{array} & \begin{array}{c}\text { no. of } \\ \text { frames }\end{array} & \begin{array}{c}\langle\mathrm{MSD}\rangle \\ \left(\AA^{2}\right)^{a}\end{array} & \lambda\left(\AA^{-2}\right) & \begin{array}{c}\langle\mathrm{SE}\rangle \\ (\mathrm{kcal} / \mathrm{mol})^{b}\end{array} \\ \text { pwf } & 5 & 1.4 & 20 & 3.54 & 0.65 & 2.18 \\ \text { pws } & 4 & 1.0 & 20 & 3.70 & 0.62 & 2.35\end{array}$

${ }^{a}$ Mean-square deviation of the displacement set. ${ }^{b}$ Mean standard error of free energy along the pathway.

metadynamics simulations only in the space of $s(\mathbf{R})$, and $z(\mathbf{R})$ was constrained to $z(\mathbf{R})<6 \AA^{2}$ (Table 1) and used to monitor the deviation from the RPs. This gave the possibility for the system to explore conformations different from $\mathbf{R}(t)$, however maintaining the trajectories close to the RPs.

II.E. Metadynamics Simulations. The time-independent estimate of free energy ${ }^{49} F(s)$ as a function of the PCV was determined by well-tempered metadynamics ${ }^{22,50}$ using $F(s, t)=-\frac{T+\Delta T}{\Delta T} V(s, t)$, where $V(s, t)$ is the biasing potential added to the system, and $T$ is the temperature of the simulation. The difference between the temperature of the PCV and the temperature of the simulation is denoted by $\Delta T$. The bias potential was composed of the sum of the adaptive Gaussians ${ }^{51}$ deposited along the trajectories of the PCV. To obtain $F(s)$, the free-energy profiles were reweighted by the time-dependent constant $c(t)$

$$
c(t)=\frac{1}{\beta} \ln \frac{\int \mathrm{e}^{-\beta F(s)} \mathrm{d} s}{\int \mathrm{e}^{-\beta[F(s)+V(s, t)]} \mathrm{d} s}
$$

where $\beta$ is the inverse temperature.

For a detailed description of metadynamics, we refer to several reviews. ${ }^{33,52,53}$ The metadynamics simulations were carried out in the NVT ensemble using Plumed2. ${ }^{54}$ For the metadynamics simulations, we used the following parameters: the adaptive Gaussians were used in the time domain and the width of the
Gaussians was $0.25 \mathrm{ps}$. The rate of the Gaussian deposition was $1.92 \mathrm{kcal} / \mathrm{mol}$ per ps. The temperature was $300 \mathrm{~K}$ and the $\Delta T$ was $3600 \mathrm{~K}$.

II.F. Rate Estimation. Under the assumption that the dynamics is Markovian, the diffusion coefficient $D(s)$ that describes the local dynamics along the free-energy profile can be estimated for any lag time $\Delta t$ and any time $t$ from $\mathbf{P}(t+\Delta t)=\mathrm{e}^{\mathrm{T} \Delta t} \mathbf{P}(t)$, where $\mathbf{T}$ is a rate matrix. ${ }^{55}$ This indicates that the likelihood of finding a trajectory at $j$ at time $t+\Delta t$, given it was at $i$ at time $t$, is proportional to $\left(\mathrm{e}^{\mathrm{T} \Delta t}\right)_{i j}$. Assuming the free-energy $F(s)$ is known, $D(s)$ can be estimated using MLE. ${ }^{55}$ Here, the rate matrix $\mathbf{T}$ was sampled by a Monte Carlo algorithm to maximize a log-likelihood,

$$
l=\sum_{i=1}^{N} \sum_{j=1}^{N} N_{i j} \ln \left(\mathrm{e}^{\mathrm{T} \Delta t}\right)_{i j}
$$

where the summation goes over the discretized $s$ from the eq 2 and $N_{i j}$ are elements of a transition matrix $\mathbf{N}$. Then, the diffusion coefficient along $s$ was calculated, $D\left(s_{i}\right)=\left(D_{i-1 / 2}+D_{i+1 / 2}\right) / 2$, where $D_{i \pm 1 / 2}$ is

$$
D_{i \pm 1 / 2}=\Delta s^{2} T_{i i \pm 1} \exp \left(\beta\left(F\left(s_{i}\right)-F\left(s_{i \pm 1 / 2}\right)\right)\right)
$$

where $\Delta s$ is the interval in the PCV space used to discretize $s$. The inverse transition rate $k$ was estimated by ${ }^{23,56}$

$$
k^{-1}=\sum_{i=1}^{N} \sum_{j=1}^{i} \frac{\mathrm{e}^{-\beta F\left(s_{j}\right)}}{D\left(s_{i}\right) \mathrm{e}^{-\beta F\left(s_{i}\right)}}
$$

where the summation is over the discretized PCV space starting from $s\left(\mathbf{R}_{A}\right)$ and ending at $s\left(\mathbf{R}_{B}\right)$.

Each RP was discretized in $N=20$ bins, and for each bin $202 \mathrm{~ns}$ long unbiased MD simulations were run to sample $s$. Using $20 \times 20$ unbiased trajectories for each RP, the transition matrices $\mathbf{N}$ were estimated ${ }^{57}$ and used to calculate $D(s)$. The transition matrix $\mathbf{N}$ was estimated for $\Delta t=2,4,8,16,32,64$, and 128 ps. The results showing the average rate for 100 Monte Carlo simulations for each lag time are shown in Figure S3 in the Supporting Information. Smaller lag times overestimated $k$, while lag times $\Delta t>64$ ps resulted in consistently similar rates, thus we used $\Delta t=128$ ps to estimate the diffusion constants and the rates.

\section{RESULTS AND DISCUSSION}

III.A. Accelerated Migration of the Inhibitor. As the average time scale of ligand dissociation is far exceeding time scales accessible using unbiased $\mathrm{MD}$ simulations, ${ }^{58}$ unbiased sampling of the dissociation of hupA from TcAChE would be demanding computationally. The sampling was enhanced locally along the RPs, ${ }^{21,24}$ providing information about the exit routes of TcAChE and accelerating the hupA dissociation.

The movement of hupA during its dissociation was evaluated by the distances of hupA from its starting position within the CAS of TcAChE as a function of time. Figure 2 shows the distances of hupA from its starting position over the $201 \mathrm{~ns}$ trajectories. The results of the Jenks optimization method indicate that the displacement of hupA can be divided into three stages with a GVF value (goodness of variance fit) $=0.9$. The displacement of hupA is for most of the simulation time $<8 \AA$ which corresponds to the first stage (st1) describing the fluctuations of hupA upon unbinding from the CAS. This result is in accord with a paper by $\mathrm{Xu}$ et al. which describes the fluctuations 


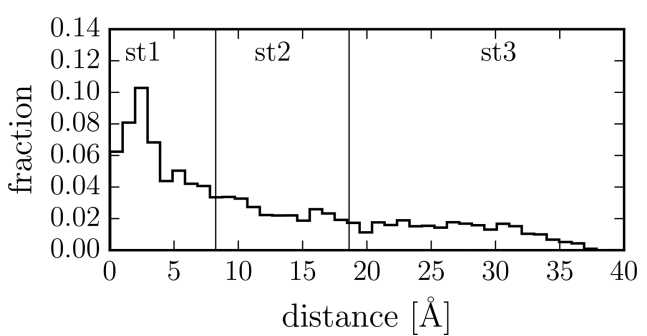

Figure 2. Histogram of the distances of hupA from its initial position within the CAS of TcAChE. The individual distances were extracted from the MS simulations. The histogram contains about 50000 data points compiled from the 0.2 ps snapshots in each of the 10 enhanced $\mathrm{MD}$ simulations of $1 \mathrm{~ns}$ duration. The distances were classified into the stages st $1-3$ based on the Jenks optimization method with a GVF value $($ goodness of variance fit $)=0.9$.

of thiocholine within the active-site gorge in a similar range of the distances. ${ }^{16}$ The second stage (st2) shows a decrease of the fraction of the distances at about 8-19 A. This stage corresponds to the migration of hupA from the CAS toward the exit of TcAChE. During st3, hupA travels in solvent. The distances corresponding to st 3 are approximately $>19 \AA$.

We also calculated the pairwise distances between the hupA conformations during dissociation. In st1 hupA is confined within the CAS which results in the mean pairwise distance of hupA conformations at about $4 \AA$. The mean pairwise distances reach higher values in st $2-10.3 \AA$ and $\mathrm{s} 3-16.4 \AA$. This means that hupA after fluctuating in the CAS (st1) starts to dissociate from TcAChE toward solvent (st2). The length of the active-site gorge leading to the CAS is about $20 \AA$ which is comparable to the distances of hupA from its initial position within TcAChE obtained during st2. Closer structural inspection shows that the last increase in the mean pairwise distances between the conformations of hupA during st 3 means that the ligand leaves the mouth of $T c A C h E$ and migrates farther in solvent. At this stage the MS simulations were terminated.

III.B. Identifying Reaction Pathways or Unbinding Intermediates. The MS simulations revealed two RPs of the hupA escape from the CAS as depicted in Figure 3. The first $\mathrm{RP}$ - pwf-involves the tunnel leading from the CAS of TcAChE via the active-site gorge, and it is accessible during the $\mathrm{MD}$ simulations (Figure $3 \mathrm{~b}$ ). No additional rearrangement within the CAS is required to open this passage for hupA. There are several studies showing the primary importance of pwf either for native products, as with tiocholine, ${ }^{16}$ or for inhibitors used in the treatment of Alzheimer's disease as with hupA. ${ }^{3,14,59}$ The second RP (pws, Figure 3c) leads from the CAS to solvent via a transient channel through the $\Omega$-loop, which consist of residues 67-94 (Figure 3a, magenta). The opening of pws in TcAChE has been reported for the unliganded $T c \mathrm{AChE}^{13,59}$ and the hupA-bound structure. ${ }^{14}$ It was proposed, however, that pws may serve as a channel for the flux of gaseous species to the active-site gorge. In contrast, our simulations suggest that the local fluctuations of the $\Omega$-loop enable hupA to escape via pws, providing an alternative dissociation route for enzymatic reactions within TcAChE.

Typical unbinding pathways are shown in Figure 3a for pwf (red) and pws (blue). Initially, hupA resides within the binding pocket guarded by Trp432, Trp442, and Phe330 (Figure 6) and interacts via $\pi$-stacking with Trp $84 .^{13}$ These residues block pwb which was observed in MD simulations and experiments with other ligands, ${ }^{15}$ but for hupA, there is no evidence showing that the escape of hupA may occur via pwb.

To study the hindrance of $T c A C h E$, we calculated the rootmean-square fluctuation (rmsf) values for the $T c A C h E$ residues averaged over the dissociation trajectories (Figure 4). There are several residues of $T c \mathrm{AChE}$ that indicate potential hindrance. The escape via pwf involves a passage near Tyr334 and Phe330 at the beginning of st2 (Figure 6). The bottleneck consisting of these aromatic residues restricts the access to the CAS.
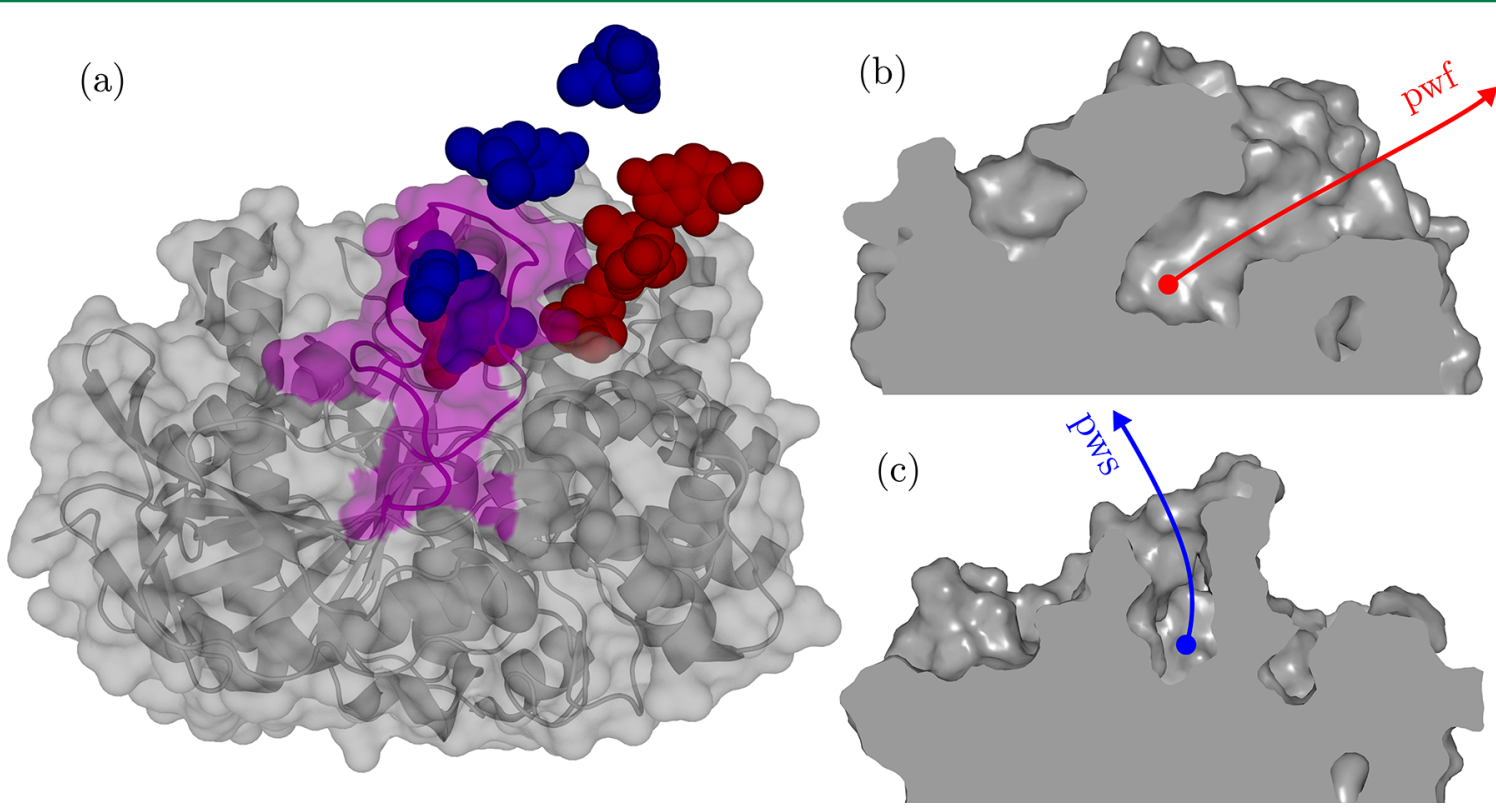

Figure 3. Ligand dissociation pathways selected as the RPs revealed by the MS simulations within TcAChE (gray). (a) Here we highlight the hupA unbinding pathways from the CAS of TcAChE through the front-exit pwf (red) and the transiently opened side-exit pws (blue), both incorporating the fluctuations of the $\Omega$-loop consisting of residues 67-94 (magenta). (b) pwf does not require large motions of TcAChE because the channel is already accessible from the beginning of the MD simulations. (c) In contrast, hupA cannot escape via the transiently opened tunnel pws unless the fluctuations of the TcAChE structure, particularly the $\Omega$-loop, are triggered. 


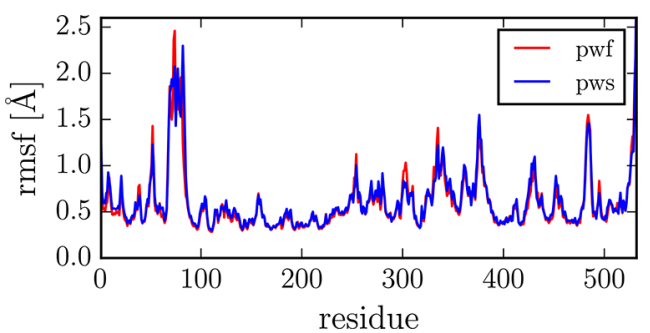

Figure 4. Root-mean-square fluctuation values of the TcAChE residues from the MS simulations shown for pwf (red) and pws (blue).

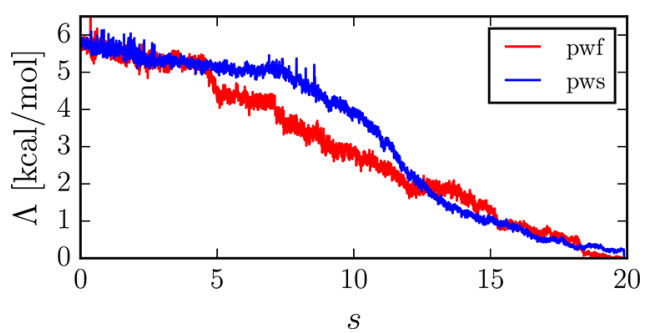

Figure 5. Effective interaction energy between the inhibitor and the enzyme minimized during the MS simulations shown for pwf (red) and pws (blue).

In particular, Phe330 has been shown experimentally to act as a gating mechanism limiting access to the CAS from the peripheral anionic site. ${ }^{60-62}$

The rmsf values are similar for both RPs. The $\Omega$-loop is the most hindered part of TcAChE (approximately $2 \AA$ ), which suggests that the deformations induced by the dissociating hupA may be similar during the exit via pwf and pws. Namely, Asp72 (rmsf at about $2.5 \AA$ ) and Trp84 accept the hydrogen atoms provided by hupA during the dissociation via pwf and pws, anchoring the inhibitor to the $\Omega$-loop (Figure S2).
We observed that Asp72, Trp84, and Asn85 are the most frequent amino acids which accept hydrogen atoms from hupA (Figure S2), which means that the $\Omega$-loop (residues 67-94) participates in the stabilization of hupA in the initial stage of unbinding. Upon dissociation, hupA is subsequently anchored by Gly80, which acts as an intermediate frequently pairing with hupA, and the hupA pyridone group accepts a hydrogen from Tyr70 (Figure S2). Moreover, Phe330 interacts with the pyridone group of hupA via $\pi$-stacking (Figure 6 ). For the profiling of hydrogen bond donor-acceptor pairs, see Figure S2 in the Supporting Information, and for the clustering of amino acids, which pair with hupA by hydrogen bonds, into stages (bound, transition, and unbound), see Figure S2 and Table S2 in the Supporting Information.

During the dissociation along pws, hupA perturbs Ser81 (rmsf at about $2.3 \AA$ ), which stabilizes the $\Omega$-loop and pairs via a hydrogen bond with hupA (Figure S2). Our results show that the rotation of hupA (Figure 6) is important for the dissociation along pws. Initially, hupA is oriented in such a way that its pyridone oxygen points toward the residues that make up the beginning and the end of the $\Omega$-loop, and the pyridone group of hupA is perpendicular to the $\Omega$-loop plane. Upon dissociation along pws, the pyridone group rotates about $60^{\circ}$ toward the $\Omega$-loop plane while preserving the orthogonality of the $\Omega$-loop plane and the pyridone group of hupA. This rotation enables passing through the $\Omega$-loop to solvent which is shown, in contrast to pwf, by a sudden decrease in the effective interaction energy of hupA-TcAChE at $s \approx 12$ (Figure 5).

III.C. Free Energy along the Reaction Pathways. To calculate free energies along the RPs, using metadynamics we biased the $s$ variable which describes the progress along the RPs. The time-independent estimator of free energy $F(s)$ was used to determine the local quality of convergence across the biased space. Additionally, the convergence of the metadynamics simulations required a decrease in the Gaussian height below $1 \%$

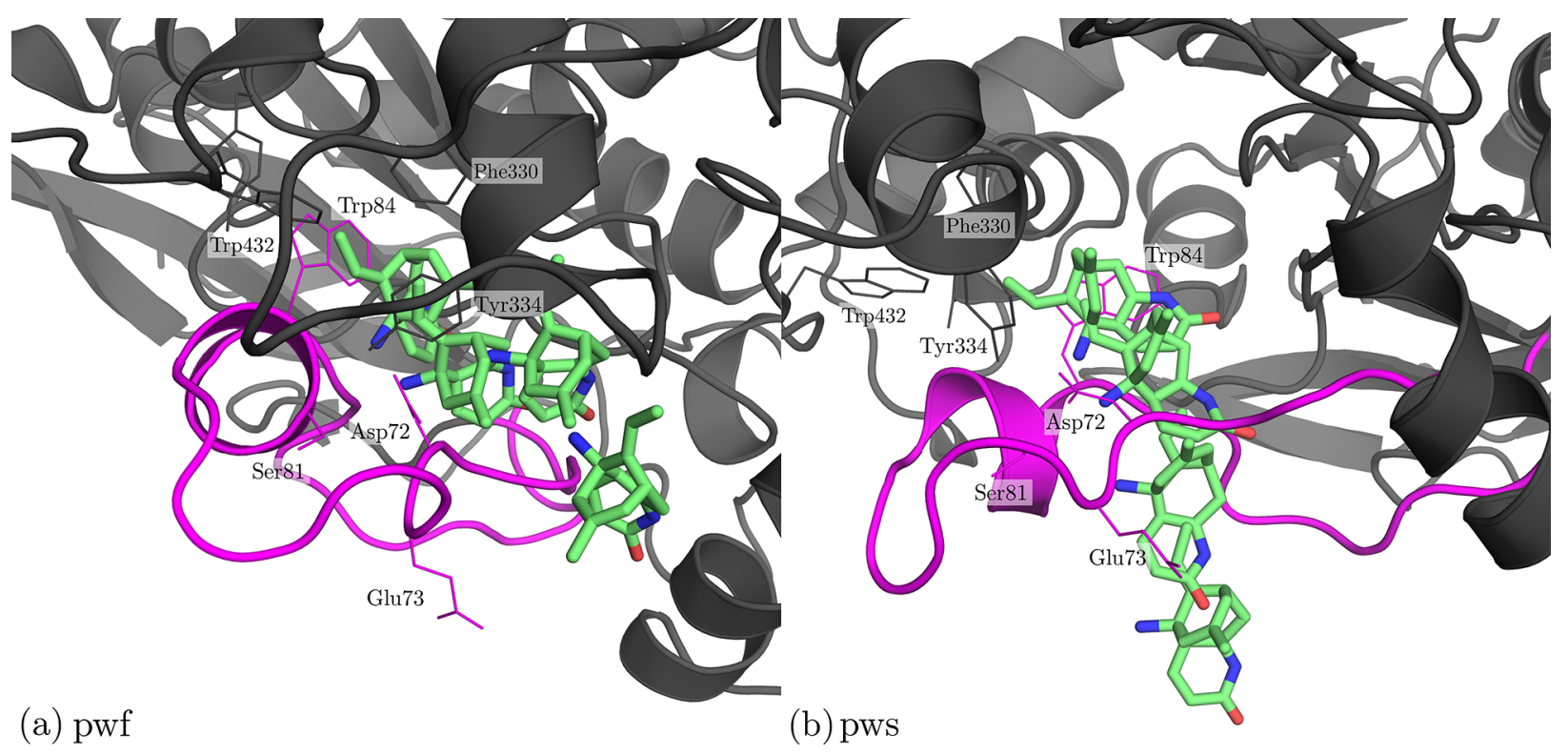

Figure 6. Structural representation of intermediate unbinding configurations along the RPs which atomistically characterize the dissociation of hupA (shown as sticks) along (a) pwf and (b) pws. In the initial stage, hupA interacts mainly with Trp84 (parallel-displaced $\pi$-stacking). Then, hupA starts to move toward solvent either via the main gorge (pwf) or through the partially disordered $\Omega$-loop (pws). The shown poses of hupA represent the local minima of free energy along the RPs (Figure 7). The orientation of hupA along pwf is unchanged during migration. In contrast, upon threading through the $\Omega$-loop (residues 67-94, purple) along pws, it can be observed that the pyridone group of the inhibitor rotates about $60^{\circ}$ toward the $\Omega$-loop plane. 
of its initial value after $200 \mathrm{~ns}$ for each RP. ${ }^{49}$ By this time, we observed multiple inhibitor recrossings (binding and unbinding events) along the sampled RPs. The standard error in the free energy shown in Figure 7 was estimated from the free-energy

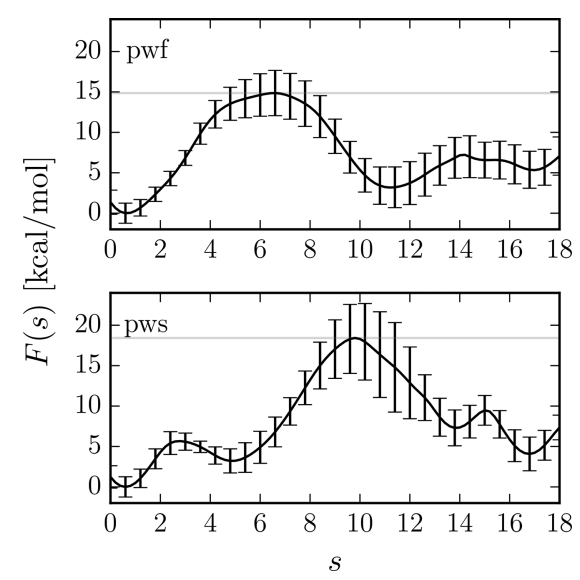

Figure 7. Free-energy profiles $F(s)$ along hupA dissociation pathways pwf and pws. The error bars are calculated as standard errors from the well-tempered metadynamics simulations. For details, see Table 1.

profiles calculated by independent metadynamics simulations (Table 1).

Figure 7 shows the free-energy profiles $F(s)$ along pwf and pws. The highest free-energy barrier along pwf exists between the first and second intermediates $(\sim 15 \mathrm{kcal} / \mathrm{mol})$, near Tyr334 and Asp72. At this stage, Glu73 rotates in the direction of migrating hupA (Figure 6a). Figure $6 \mathrm{~b}$ shows the hupA configurations threading through the $\Omega$-loop corresponding to the dissociation along pws. The highest free-energy barrier along pws is about $18 \mathrm{kcal} / \mathrm{mol}$, which is similar to that of pwf. This was likely due to the rotation of hupA which occurs before hupA threads through the $\Omega$-loop (Figure $6 \mathrm{~b}$ ), enabling an alternative egress route. This mechanism is particularly pronounced in TcAChE since the partially disordered $\Omega$-loop allows for an easy structural adjustment to the inhibitor.

The second intermediate along pws $(s=5)$ lies within a metastable free-energy well $(\sim 4 \mathrm{kcal} / \mathrm{mol})$ which attracts the trajectories before piercing the $\Omega$-loop (Figure 6). Next, pws opens because it is transiently triggered by the enforced inhibitor dissociation. Several minima corresponding to the transition states of the unbinding are located along pwf and pws before the complex reaches the dissociated configuration (Figure 7). Those minima translate to thermodynamically stable poses along the RPs, as shown in Figure 6. The free-energy profiles along pws and pwf show a comparable number of intermediates with the free-energy barriers similar within the error bars (Figure 7). In systems with multiple unbinding pathways in which the transition states are comparable in free energy, perturbations to ligandenzyme interactions could potentially shift which pathway is the dominant dissociation pathway. Our simulations show that pathway hopping, as termed by Lotz and Dickson, ${ }^{63}$ can be observed for TcAChE inhibited by hupA. This phenomenon was also noticed in the context of protein folding. ${ }^{64}$

III.D. Dissociation Kinetics. As we showed, the $\Omega$-loop modulates the hupA dissociation by providing access to pwf or another relevant unbinding pathway such as pws, which may advance the understanding of the slow-onset inhibition in this instance. We estimated the rates along the RPs based on $2 \times$ $800 \mathrm{~ns}$ of the unbiased MD simulations (Section II.F and
Section S3). From these simulations, we calculated the transition matrix for the lag time $\Delta t=128$ ps (Figure S3) which was used to estimate the diffusion coefficients $D(s)$ along pwf and pws by Monte Carlo simulations (Figure S4). The rates were calculated for the averaged free-energy profiles using eq 7. In the used method, microkinetics of interstate transitions between intermediates along both RPs is combined to estimate the rates, which is similar to hyperdynamics. ${ }^{65}$ For details concerning the procedure applied to compute the rates and the errors in the rates along both RPs, see the Supporting Information (Section S3).

The estimated mean-first-passage time (the inverse rate) along the main gorge (pwf) of the enzyme was about $10 \mathrm{~min}$, which agrees with a residence time measured experimentally $(24 \mathrm{~min}) .{ }^{66}$ This translates to a rate of $(12.5 \pm 9) \times 10^{-3} \mathrm{~s}^{-1}$, indicating that the time scale of the hupA dissociation is similar to that of slow-onset inhibition. ${ }^{18,67}$ Our results of the kinetic rate along pwf agree with rates determined from surface plasmon resonance experiments ${ }^{13}$ (i.e., $1.8 \times 10^{-3}-8.01 \times 10^{-3} \mathrm{~s}^{-1}$ ). The kinetic rate along pws was $(2.1 \pm 1.4) \times 10^{-3} \mathrm{~s}^{-1}$, showing that the dissociation of hupA from TcAChE is possible along the multiple RPs chosen by the inhibitor. The kinetic rate calculated along pws is similar to that along pwf. The meanfirst-passage time along pws is approximately equal to $19 \mathrm{~min}$. We identified the fluctuations of the $\Omega$-loop and the orientation of the hupA pyridone group relative to the $\Omega$-loop plane as possible reasons for the comparable dissociation times along both unbinding pathways.

On the basis of the kinetic results, we hypothesize that apart from the deviation of Phe330 and Tyr334 observed in experiments ${ }^{60-62}$ the transient opening of pws caused by the orientation of hupA near the $\Omega$-loop may play a structural role in providing another relevant dissociation pathway, which may be important for the slow-onset inhibition of TcAChE. This is characterized by an intermediate of hupA and the $\Omega$-loop that helps to open pws, resulting in a free-energy barrier of the dissociation process similar to that of pwf. This provides a more detailed picture of the slow-onset inhibition of TcAChE than was previously speculated and suggests that, given the free energies and the rates, the inhibition of TcAChE could also operate along pws.

\section{CONCLUSIONS}

Investigating enzyme-inhibitor dissociation in terms of thermodynamics and kinetics is important in neuropharmacology and medical biochemistry. ${ }^{68}$ Accounting for protein internal motions in the calculating of physical reaction coordinates of ligand dissociation is necessary. ${ }^{33,69-71}$ Here we researched the slow-onset inhibition of Torpedo californica AChE by the hupA inhibitor. Because slow-onset inhibition occurs on time scales that span from seconds to hours, our approach required statistical mechanics methods comprising mainly biased sampling of the RPs by MS and well-tempered metadynamics to reconstruct free-energy barriers along the RPs. During the well-tempered metadynamics simulations, the pathways were sampled in both directions (i.e., dissociation and association), resulting in multiple recrossings of hupA along the RPs. Moreover, additional unbiased $\mathrm{MD}$ simulations were used to estimate the kinetic rates along the RPs.

We uncovered two RPs: a front door along the axis of the active-site gorge (pwf), previously analyzed in a study by $\mathrm{Xu}$ et al., ${ }^{3}$ and through a new transient side door (pws), i.e., the $\Omega$-loop (residues 67-94 of TcAChE) that was not proposed as 
a channel for inhibitors. The corresponding free-energy profiles and kinetic rates illustrated that the main rate-limiting step comes from a structural bottleneck near Phe330 and Tyr334, which was also observed experimentally, ${ }^{60-62}$ and the fluctuations of the $\Omega$-loop. The kinetic rates estimated along the RPs agreed with available experimental data from surface plasmon resonance. $^{13}$

The free-energy barriers of the hupA dissociation from the active gorge along pwf and pws imply that the kinetic rates estimated along these pathways are similar and within the regime of time scales observed experimentally for slow-onset inhibition. The transient opening of the $\Omega$-loop along pws could act as an alternative dissociation route for inhibitors, showing that the inhibition mechanism of $\mathrm{TcAChE}$ can operate interchangeably along the two RPs. In agreement with a study by Bui et al., 72 we conclude that displacements of the $\Omega$-loop favor alternative dissociation routes from the active site of the enzyme. The transient opening of the partially disordered $\Omega$-loop could be concurrent with the dissociation along pwf. In conclusion, a complex interplay of the different unbinding pathways and intermediates (of similar free energies) leading to pathway hopping ${ }^{63,64}$ could be important for the slow-onset inhibition of TcAChE by hupA. This information may provide the molecular basis for the rational design of next-generation inhibitors for the treatment of Alzheimer's disease with increased residence time.

\section{ASSOCIATED CONTENT}

\section{S Supporting Information}

The Supporting Information is available free of charge on the ACS Publications website at DOI: 10.1021/acs.jctc.8b00173.

MD simulations and corresponding simulation times. Hydrogen donor-acceptor pairs between hupA and the enzyme. Paired residues and their Euclidean distance to the initial conformation of hupA. MLE protocol used to calculate the kinetic rates along pwf and pws, including exemplary transition matrixes for the lag time $\Delta t=2 \mathrm{ps}$, the acceptance ratio of the Monte Carlo protocol, and the errors in the kinetic rates calculated for different values of the lag time. (PDF)

\section{AUTHOR INFORMATION}

\section{Corresponding Author}

*E-mail: jr@fizyka.umk.pl.

\section{ORCID}

J. Rydzewski: 0000-0003-4325-4177

Notes

The authors declare no competing financial interest.

\section{ACKNOWLEDGMENTS}

J.R. acknowledges funding (grant 2016/20/T/ST3/00488) from the National Science Centre, Poland. This work was performed while J.R. was a guest of the Max Planck Institute for Biophysical Chemistry in Göttingen. R.J. acknowledges support from the Polish Ministry for Science and Higher Education Grant 0003/ID3/2016/64 (Ideas Plus II). The results were obtained at the Interdisciplinary Centre for Modern Technologies, NCU.

\section{REFERENCES}

(1) Shen, T.; Tai, K.; Henchman, R. H.; McCammon, J. A. Molecular dynamics of acetylcholinesterase. Acc. Chem. Res. 2002, 35, 332-340.
(2) Colletier, J.-P.; Fournier, D.; Greenblatt, H. M.; Stojan, J.; Sussman, J. L.; Zaccai, G.; Silman, I.; Weik, M. Structural insights into substrate traffic and inhibition in acetylcholinesterase. EMBO J. 2006 , $25,2746-2756$.

(3) Xu, Y.; Shen, J.; Luo, X.; Silman, I.; Sussman, J. L.; Chen, K.; Jiang, $\mathrm{H}$. How does huperzine $\mathrm{A}$ enter and leave the binding gorge of acetylcholinesterase? Steered molecular dynamics simulations. J. Am. Chem. Soc. 2003, 125, 11340-11349.

(4) Silman, I.; Sussman, J. L. Acetylcholinesterase: 'classical' and 'non-classical' functions and pharmacology. Curr. Opin. Pharmacol. 2005, 5, 293-302.

(5) Laganiere, S.; Corey, J.; Tang, X.-C.; Wülfert, E.; Hanin, I. Acute and chronic studies with the anticholinesterase huperzine A: effect on central nervous system cholinergic parameters. Neuropharmacology 1991, 30, 763-768.

(6) Sussman, J. L.; Harel, M. Atomic structure of acetylcholinesterase from Torpedo californica: a prototypic acetylcholine-binding protein. Science 1991, 253, 872-879.

(7) Kaur, J.; Zhang, M. Molecular modelling and QSAR of reversible acetylcholinesterase inhibitors. Curr. Med. Chem. 2000, 7, 273-294.

(8) Quinn, D. M. Acetylcholinesterase: enzyme structure, reaction dynamics, and virtual transition states. Chem. Rev. 1987, 87, 955-979.

(9) Raves, M. L.; Harel, M.; Pang, Y.-P.; Silman, I.; Kozikowski, A. P.; Sussman, J. L. Structure of acetylcholinesterase complexed with the nootropic alkaloid,(-)-huperzine A. Nat. Struct. Mol. Biol. 1997, 4, $57-63$.

(10) Camps, P.; El Achab, R.; Morral, J.; Muñoz-Torrero, D.; Badia, A.; Baños, J. E.; Vivas, N. M.; Barril, X.; Orozco, M.; Luque, F. J. New tacrine- huperzine A hybrids (huprines): highly potent tight-binding acetylcholinesterase inhibitors of interest for the treatment of Alzheimer's disease. J. Med. Chem. 2000, 43, 4657-4666.

(11) Ferreira, A.; Rodrigues, M.; Fortuna, A.; Falcão, A.; Alves, G. Huperzine A from Huperzia serrata: a review of its sources, chemistry, pharmacology and toxicology. Phytochem. Rev. 2016, 15, 51-85.

(12) Dvir, H.; Jiang, H.; Wong, D.; Harel, M.; Chetrit, M.; He, X.; Jin, G.; Yu, G.; Tang, X.; Silman, I. X-ray structures of Torpedo californica acetylcholinesterase complexed with (+)-huperzine A and (-)-huperzine B: Structural evidence for an active site rearrangement. Biochemistry 2002, 41, 10810-10818.

(13) Bai, F.; Xu, Y.; Chen, J.; Liu, Q.; Gu, J.; Wang, X.; Ma, J.; Li, H.; Onuchic, J. N.; Jiang, H. Free energy landscape for the binding process of Huperzine a to acetylcholinesterase. Proc. Natl. Acad. Sci. U. S. A. 2013, 110, 4273-4278.

(14) Tara, S.; Helms, V.; Straatsma, T.; McCammon, J. A. Molecular dynamics of mouse acetylcholinesterase complexed with huperzine $\mathrm{A}$. Biopolymers 1999, 50, 347-359.

(15) Sanson, B.; Colletier, J.-P.; Xu, Y.; Lang, P. T.; Jiang, H.; Silman, I.; Sussman, J. L.; Weik, M. Backdoor opening mechanism in acetylcholinesterase based on X-ray crystallography and molecular dynamics simulations. Protein Sci. 2011, 20, 1114-1118.

(16) Xu, Y.; Colletier, J.-P.; Weik, M.; Qin, G.; Jiang, H.; Silman, I.; Sussman, J. L. Long route or shortcut? a molecular dynamics study of traffic of thiocholine within the active-site gorge of acetylcholinesterase. Biophys. J. 2010, 99, 4003-4011.

(17) Masson, P.; Lushchekina, S. V. Slow-binding inhibition of cholinesterases, pharmacological and toxicological relevance. Arch. Biochem. Biophys. 2016, 593, 60-68.

(18) Li, H.-J.; Lai, C.-T.; Pan, P.; Yu, W.; Liu, N.; Bommineni, G. R.; Garcia-Diaz, M.; Simmerling, C.; Tonge, P. J. A structural and energetic model for the slow-onset inhibition of the Mycobacterium tuberculosis enoyl-ACP reductase InhA. ACS Chem. Biol. 2014, 9, 986-993.

(19) De Vivo, M.; Masetti, M.; Bottegoni, G.; Cavalli, A. Role of molecular dynamics and related methods in drug discovery. J. Med. Chem. 2016, 59, 4035-4061.

(20) Bruce, N. J.; Ganotra, G. K.; Kokh, D. B.; Sadiq, S. K.; Wade, R. C. New approaches for computing ligand-receptor binding kinetics. Curr. Opin. Struct. Biol. 2018, 49, 1-10. 
(21) Rydzewski, J.; Nowak, W. Memetic algorithms for ligand expulsion from protein cavities. J. Chem. Phys. 2015, 143, 124101.

(22) Barducci, A.; Bussi, G.; Parrinello, M. Well-tempered metadynamics: a smoothly converging and tunable free-energy method. Phys. Rev. Lett. 2008, 100, 020603.

(23) Singharoy, A.; Chipot, C.; Moradi, M.; Schulten, K. Chemomechanical coupling in hexameric protein-protein interfaces harnesses energy within v-Type ATPases. J. Am. Chem. Soc. 2017, 139, 293-310.

(24) Rydzewski, J.; Nowak, W. Machine learning based dimensionality reduction facilitates ligand diffusion paths assessment: a case of cytochrome P450cam. J. Chem. Theory Comput. 2016, 12, 2110-2120.

(25) Rydzewski, J.; Nowak, W. Thermodynamics of camphor migration in cytochrome P450cam by atomistic simulations. Sci. Rep. 2017, 7, 7736

(26) Pietrucci, F.; Marinelli, F.; Carloni, P.; Laio, A. Substrate binding mechanism of HIV-1 protease from explicit-solvent atomistic simulations. J. Am. Chem. Soc. 2009, 131, 11811-11818.

(27) Paloncýová, M.; Navrátilová, V.; Berka, K.; Laio, A.; Otyepka, $M$. Role of enzyme flexibility in ligand access and egress to active site: Bias-exchange metadynamics study of 1, 3, 7-trimethyluric acid in cytochrome P450 3A4. J. Chem. Theory Comput. 2016, 12, 2101-2109.

(28) Wang, Y.; Papaleo, E.; Lindorff-Larsen, K. Mapping transiently formed and sparsely populated conformations on a complex energy landscape. eLife 2016, 5, e17505.

(29) Fidelak, J.; Juraszek, J.; Branduardi, D.; Bianciotto, M.; Gervasio, F. L. Free-energy-based methods for binding profile determination in a congeneric series of cdk2 inhibitors. J. Phys. Chem. B 2010, 114, 95169524.

(30) Saladino, G.; Gauthier, L.; Bianciotto, M.; Gervasio, F. Assessing the performance of metadynamics and path variables in predicting the binding free energies of p38 inhibitors. J. Chem. Theory Comput. 2012, $8,1165-1170$

(31) Provasi, D.; Artacho, M. C.; Negri, A.; Mobarec, J. C.; Filizola, M. Ligand-induced modulation of the free-energy landscape of $G$ protein-coupled receptors explored by adaptive biasing techniques. PLoS Comput. Biol. 2011, 7, e1002193.

(32) Furini, S.; Domene, C. Computational studies of transport in ion channels using metadynamics. Biochim. Biophys. Acta, Biomembr. 2016, 1858, 1733-1740.

(33) Rydzewski, J.; Nowak, W. Ligand diffusion in proteins via enhanced sampling in molecular dynamics. Phys. Life Rev. 2017, 22-23, 58.

(34) Jacobson, M. P.; Pincus, D. L.; Rapp, C. S.; Day, T. J.; Honig, B.; Shaw, D. E.; Friesner, R. A. A hierarchical approach to all-atom protein loop prediction. Proteins: Struct., Funct., Genet. 2004, 55, 351-367.

(35) Vanommeslaeghe, K.; MacKerell, A. D., Jr Automation of the CHARMM general force field (CGENFF) i: bond perception and atom typing. J. Chem. Inf. Model. 2012, 52, 3144-3154.

(36) Vanommeslaeghe, K.; Raman, E. P.; MacKerell, A. D., Jr Automation of the CHARMM General force field (CGENFF) ii: assignment of bonded parameters and partial atomic charges. J. Chem. Inf. Model. 2012, 52, 3155-3168.

(37) Mayne, C. G.; Saam, J.; Schulten, K.; Tajkhorshid, E.; Gumbart, J. C. Rapid parameterization of small molecules using the force field toolkit. J. Comput. Chem. 2013, 34, 2757-2770.

(38) Frisch, M. J.; Schlegel, H. B.; Scuseria, G. E.; Robb, M. A.; Cheeseman, J. R.; Scalmani, G.; Barone, V.; Mennucci, B.; Petersson, G. A.; Nakatsuji, H.; Caricato, M.; Li, X.; Hratchian, H. P.; Izmaylov, A. F.; Bloino, J.; Zheng, G.; Sonnenberg, J. L.; Hada, M.; Ehara, M.; Toyota, K.; Fukuda, R.; Hasegawa, J.; Ishida, M.; Nakajima, T.; Honda, Y.; Kitao, O.; Nakai, H.; Vreven, T.; Montgomery, J. A.; Peralta, J. E.; Ogliaro, F.; Bearpark, M.; Heyd, J. J.; Brothers, E.; Kudin, K. N.; Staroverov, V. N.; Kobayashi, R.; Normand, J.; Raghavachari, K.; Rendell, A.; Burant, J. C.; Iyengar, S. S.; Tomasi, J.; Cossi, M.; Rega, N.; Millam, J. M.; Klene, M.; Knox, J. E.; Cross, J. B.; Bakken, V.; Adamo, C.; Jaramillo, J.; Gomperts, R.; Stratmann, R. E.; Yazyev, O.; Austin, A. J.; Cammi, R.; Pomelli, C.; Ochterski, J. W.; Martin, R. L.; Morokuma, K.; Zakrzewski, V. G.; Voth, G. A.; Salvador, P.;
Dannenberg, J. J.; Dapprich, S.; Daniels, A. D.; Farkas; Foresman, J. B.; Ortiz, J. V.; Cioslowski, J.; Fox, D. J. Gaussian 09, Revision B.01, 2009.

(39) MacKerell, A. D., Jr; Bashford, D.; Bellott, M.; Dunbrack, R. L., Jr; Evanseck, J. D.; Field, M. J.; Fischer, S.; Gao, J.; Guo, H.; Ha, S. Allatom empirical potential for molecular modeling and dynamics studies of proteins. J. Phys. Chem. B 1998, 102, 3586-3616.

(40) Vanommeslaeghe, K.; Hatcher, E.; Acharya, C.; Kundu, S.; Zhong, S.; Shim, J.; Darian, E.; Guvench, O.; Lopes, P.; Vorobyov, I. CHARMM general force field: A force field for drug-like molecules compatible with the CHARMM all-atom additive biological force fields. J. Comput. Chem. 2010, 31, 671-690.

(41) Friesner, R. A.; Banks, J. L.; Murphy, R. B.; Halgren, T. A.; Klicic, J. J.; Mainz, D. T.; Repasky, M. P.; Knoll, E. H.; Shelley, M.; Perry, J. K. Glide: a new approach for rapid, accurate docking and scoring. 1. method and assessment of docking accuracy. J. Med. Chem. 2004, 47, 1739-1749.

(42) Feller, S. E.; Zhang, Y.; Pastor, R. W.; Brooks, B. R. Constant pressure molecular dynamics simulation: the Langevin piston method. J. Chem. Phys. 1995, 103, 4613-4621.

(43) Phillips, J. C.; Braun, R.; Wang, W.; Gumbart, J.; Tajkhorshid, E.; Villa, E.; Chipot, C.; Skeel, R. D.; Kale, L.; Schulten, K. Scalable molecular dynamics with namd. J. Comput. Chem. 2005, 26, 17811802.

(44) Jorgensen, W. L.; Chandrasekhar, J.; Madura, J. D.; Impey, R. W.; Klein, M. L. Comparison of simple potential functions for simulating liquid water. J. Chem. Phys. 1983, 79, 926-935.

(45) Darden, T.; York, D.; Pedersen, L. Particle mesh Ewald: An NlogN method for ewald sums in large systems. J. Chem. Phys. 1993, 98, 10089-10092.

(46) Michaud-Agrawal, N.; Denning, E. J.; Woolf, T. B.; Beckstein, O. Mdanalysis: a toolkit for the analysis of molecular dynamics simulations. J. Comput. Chem. 2011, 32, 2319-2327.

(47) Branduardi, D.; Gervasio, F. L.; Parrinello, M. From a to b in free energy space. J. Chem. Phys. 2007, 126, 054103.

(48) Limongelli, V.; Bonomi, M.; Marinelli, L.; Gervasio, F. L.; Cavalli, A.; Novellino, E.; Parrinello, M. Molecular basis of cyclooxygenase enzymes (COXs) selective inhibition. Proc. Natl. Acad. Sci. U. S. A. 2010, 107, 5411-5416.

(49) Tiwary, P.; Parrinello, M. A time-independent free energy estimator for metadynamics. J. Phys. Chem. B 2015, 119, 736-742.

(50) Laio, A.; Parrinello, M. Escaping free-energy minima. Proc. Natl. Acad. Sci. U. S. A. 2002, 99, 12562-12566.

(51) Branduardi, D.; Bussi, G.; Parrinello, M. Metadynamics with adaptive gaussians. J. Chem. Theory Comput. 2012, 8, 2247-2254.

(52) Laio, A.; Gervasio, F. L. Metadynamics: a method to simulate rare events and reconstruct the free energy in biophysics, chemistry and material science. Rep. Prog. Phys. 2008, 71, 126601.

(53) Valsson, O.; Tiwary, P.; Parrinello, M. Enhancing important fluctuations: Rare events and metadynamics from a conceptual viewpoint. Annu. Rev. Phys. Chem. 2016, 67, 159-184.

(54) Tribello, G. A.; Bonomi, M.; Branduardi, D.; Camilloni, C.; Bussi, G. Plumed 2: New feathers for an old bird. Comput. Phys. Commun. 2014, 185, 604-613.

(55) Hummer, G. Position-dependent diffusion coefficients and free energies from bayesian analysis of equilibrium and replica molecular dynamics simulations. New J. Phys. 2005, 7, 34.

(56) Szabo, A.; Schulten, K.; Schulten, Z. First passage time approach to diffusion controlled reactions. J. Chem. Phys. 1980, 72, 4350-4357.

(57) Best, R.; Hummer, G. Diffusion models of protein folding. Phys. Chem. Chem. Phys. 2011, 13, 16902-16911.

(58) Núñez, S.; Venhorst, J.; Kruse, C. G. Target-drug interactions: first principles and their application to drug discovery. Drug Discovery Today 2012, 17, 10-22.

(59) Tara, S.; Straatsma, T.; McCammon, J. A. Mouse acetylcholinesterase unliganded and in complex with huperzine A: A comparison of molecular dynamics simulations. Biopolymers 1999, 50, $35-43$. 
(60) Kryger; Silman, I.; Sussman, J. L. Structure of acetylcholinesterase complexed with E2020 (Aricept): implications for the design of new anti-alzheimer drugs. Structure 1999, 7, 297-307.

(61) Harel, M.; Schalk, I.; Ehret-Sabatier, L.; Bouet, F.; Goeldner, M.; Hirth, C.; Axelsen, P.; Silman, I.; Sussman, J. Quaternary ligand binding to aromatic residues in the active-site gorge of acetylcholinesterase. Proc. Natl. Acad. Sci. U. S. A. 1993, 90, 9031-9035.

(62) Xu, Y.; Colletier, J.-P.; Weik, M.; Jiang, H.; Moult, J.; Silman, I.; Sussman, J. L. Flexibility of aromatic residues in the active-site gorge of acetylcholinesterase: X-ray versus molecular dynamics. Biophys. J. 2008, 95, 2500-2511.

(63) Lotz, S. D.; Dickson, A. Unbiased molecular dynamics of $11 \mathrm{~min}$ timescale drug unbinding reveals transition state stabilizing interactions. J. Am. Chem. Soc. 2018, 140, 618-628.

(64) Butler, J. S.; Loh, S. N. Kinetic partitioning during folding of the p53 dna binding domain. J. Mol. Biol. 2005, 350, 906-918.

(65) Schneider, J.; Reuter, K. Efficient calculation of microscopic dissolution rate constants: The aspirin-water interface. J. Phys. Chem. Lett. 2014, 5, 3859-3862.

(66) Ashani, Y.; Peggins, J. O.; Doctor, B. P. Mechanism of inhibition of cholinesterases by huperzine A. Biochem. Biophys. Res. Commun. 1992, 184, 719-726.

(67) Schramm, V. L. Enzymatic transition states, transition-state analogs, dynamics, thermodynamics, and lifetimes. Annu. Rev. Biochem. 2011, 80, 703-732.

(68) Amaral, M.; Kokh, D.; Bomke, J.; Wegener, A.; Buchstaller, H.; Eggenweiler, H.; Matias, P.; Sirrenberg, C.; Wade, R.; Frech, M. Protein conformational flexibility modulates kinetics and thermodynamics of drug binding. Nat. Commun. 2017, 8, 2276.

(69) Huang, Y.-m. M.; Raymundo, M. A. V.; Chen, W.; Chang, C.-e. A. Mechanism of the association pathways for a pair of fast and slow binding ligands of HIV-1 protease. Biochemistry 2017, 56, 1311-1323.

(70) Stank, A.; Kokh, D. B.; Fuller, J. C.; Wade, R. C. Protein binding pocket dynamics. Acc. Chem. Res. 2016, 49, 809-815.

(71) Gora, A.; Brezovsky, J.; Damborsky, J. Gates of enzymes. Chem. Rev. 2013, 113, 5871-5923.

(72) Bui, J. M.; Tai, K.; McCammon, J. A. Acetylcholinesterase: enhanced fluctuations and alternative routes to the active site in the complex with fasciculin-2. J. Am. Chem. Soc. 2004, 126, 7198-7205. 